\title{
Radionuclide Analysis Using Solid Phase Extraction Disks (U)
}

by

D. M Beals

Westinghouse Savannah River Company

Savannah River Site

Aiken, South Carolina 29808

W. G. Britt

J. P. Bibler

D. A Brooks

A document prepared for METHODS AND APPLICATIONS OF RADIOANALYTICAL CHEMISTRY CONFERENCE JOURNAL OF RADIOANALYTICAL AND NUCLEAR CHEMISTRY at Kona, HI, USA from 4/6/97 - 4/11/97.

DOE Contract No. DE-AC09-89SR18035 \& $\triangle E-A C 09-965 R / 8500$

This paper was prepared in connection with work done under the above contract number with the U.S.

Department of Energy. By acceptance of this paper, the publisher and/or recipient acknowledges the U.S.

Government's right to retain a nonexclusive, royalty-free license in and to any copyright covering this paper, along with the right to reproduce and to authorize others to reproduce all or part of the copyrighted paper. 


\section{DISCLAMMER}

\section{Portions of this document may be illegible in electronic image products. Images are produced from the best available original document.}




\section{DISCLAIMER}

This report was prepared as an account of work sponsored by an agency of the United States Government. Neither the United States Government nor any agency thereof, nor any of their cmployees, makes any warranty, express or implied, or assumes any legal liability or responsibility for the accuracy, completeness, or usefulness of any information, apparatus, product, or process disclosed, or represents that its use would not infringe privately owned rights. Reference herein to any specific commercial product, process, or service by trade name, trademark, manufacturer, or otherwise does not necessarily constitute or imply its endorsement, recommendation, or favoring by the United States Govermment or any agency thereof. The views and opinions of authors expressed herein do not necessarily state or reflect those of the United States Government or any agency thereof.

This report has been reproduced directly from the best available copy.

Available to DOE and DOE contractors from the Office of Scientific and Technical Information, P.O. Box 62, Oak Ridge, TN 37831; prices available from (615) 57.6-8401.

Available to the public from the National Technical Information Service, U.S. Department of Commerce, 5285 Port Royal Road, Springfield, VA 22161. 


\title{
Radionuclide Analysis Using Solid Phase Extraction Disks
}

\author{
Donna M. Beals, Wanda G. Britt, Jane P. Bibler, Dorothy A. Brooks \\ Savannah River Technology Center \\ Westinghouse Savannah River Company
}

Aiken SC 29808

\begin{abstract}
The use of solid phase extraction (SPE) disks was studied for the quantification of selected radionuclides in aqueous solutions. The extraction of four radionuclides using six types (two commercial, four test materials) of $3 \mathrm{M}$ Empore ${ }^{\mathrm{TM}} \mathrm{RAD}$ disks was studied. The radionuclides studied were: technetium-99 (two types of disks), cesium-137 (two types), strontium-90 (one type), plutonium-238 (one type). Extractions were tested from DI water, river water and seawater. Extraction efficiency, kinetics (flow rate past the disk), capacity, and potential interferences were studied as well as quantification methods.
\end{abstract}

\section{INTRODUCTION}

Methods incorporating solid phase extraction (SPE) disks for the quantification of specific radionuclides were evaluated as possible alternatives to wet chemical separations from large volumes of surface water. Monitoring for radionuclides in surface waters is required around nuclear facilities to fulfill regulatory compliance and to ensure the public safety. However, most radionuclides are present in extremely low concentrations requiring extensive processing of large water samples prior to analysis. Current environmental sampling and analysis procedures used at the Savannah River Site (SRS), a Department of Energy nuclear production facility located in Aiken SC (currently in standby), require 
the collection of large volume water samples. In the lab, samples are often processed by evaporation, selective precipitation, and ion exchange concentration prior to chemical separation in order to achieve the required detection limits and specificity for reporting to regulatory agencies. The use of selective SPE disks (3M Empore ${ }^{\mathrm{TM}}$ RAD disks) was evaluated in terms of ease of sample processing, cost effectiveness and detection limit of the analysis. The elements studied were technetium, cesium, strontium and plutonium.

Empore $^{\mathrm{TM}} \cdot \mathrm{RAD}$ disks are a combination of $3 \mathrm{M}$ Empore ${ }^{\mathrm{TM}}$ Membrane technology and selective adsorption resin technology. Empore ${ }^{\mathrm{TM}}$ disks contain chromatographic particles enmeshed in a network of PTFE fibrils to form a strong porous sheet, or membrane. The properties of the membrane are determined by the sorptive or reactive properties of the chosen particle. Initially, membranes were prepared containing hydrophobic octyl-and octadecyl-bonded silica particles for reversed-phase extractions of environmental pollutants ${ }^{1}$ and used to replace liquid-liquid extractions. This technology was then expanded to include membranes that were selective for the extraction of radionuclides from solution. The testing of the developed $\mathrm{RAD}$ disks is discussed below.

Two commercially available Empore ${ }^{\mathrm{TM}} \mathrm{RAD}$ disks as well as four other specially prepared materials were tested. The Sr RAD disk makes use of IBC Advanced Technologies, Inc. AnaLig ${ }^{\mathrm{TM}}$ Molecular Recognition Technology such that the AnaLig adsorbent particles in the disk allow selective adsorption of strontium. The Tc RAD disks contain GD-1 sorbent for the selective adsorption of technetium. Both of these are commercially available.

The remaining membranes tested were prepared only for this study. 3M Corp. prepared a membrane containing the EIChroM Industries, Inc. (Darien II) TEVA resin, which has been well characterized for the extraction of technetium from aqueous solution ${ }^{2}$. We also evaluated a disk for the 
extraction of plutonium from solution; this disk had sodium titanate incorporated in the Empore ${ }^{\mathrm{TM}}$ membrane. 3M Corp. also prepared two types of SPE disks for the extraction of Cs. One disk contained the sodium form of resorcinol-formaldehyde resin; resorcinol-formaldehyde resin has been tested and used at the SRS for the removal of Cs from waste ${ }^{3}$. The other test disk for Cs contains potassium cobalty ferrocyanide (KCFC). Hexacyanoferrate compounds have often been used for the environmental analysis of radiocesium ${ }^{4,5}$. A complete discussion of all the test results can be found in Beals, et al. ${ }^{6}$; the results of the testing of the two commercial disks (Tc RAD and Sr RAD) and the test Cs RAD disk containing KCFC are discussed below.

\section{ANALYSIS METHODS}

The extraction of technetium from DI water, filtered and unfiltered river water, and filtered seawater was studied. Standard solutions of Tc-99 were used to spike test solutions. Aliquots of spiked solutions containing Tc-99 were counted by liquid scintillation spectrometry to determine solution activity before and after passing through the SPE disk. For counting, three milliliters of solution was placed into a plastic liquid scintillation vial along with 19 milliliters of liquid scintillation cocktail; samples were counted for 30 minutes using an energy window of 4.0-300.0 KeV. The SPE disks were counted for adsorbed Tc-99 by either placing the disk in a liquid scintillation vial with $3 \mathrm{~mL}$ of DI water and $19 \mathrm{~mL}$ of cocktail, counting as above, or alternatively, the disk was placed on a two inch stainless steel holder and counted for twenty minutes by gas flow beta proportional spectrometry.

Uptake of strontium from solution was studied by spiking DI water or river water with a known amount of Sr-90. SPE disks that had been exposed to test solutions containing $\mathrm{Sr}-90$ were counted by gas flow beta proportional counting. Samples were counted for 20 minutes each. 
Solutions (DI water, river water and seawater) for testing the Cs specific SPE disk were spiked with Cs-137. The Cs-137 concentration of the solution or disk was measured by gamma spectrometry, using the $661.6 \mathrm{KeV}$ gamma ray of Cs-137 for calculation of the sample activity. Although Cs-137 is also a beta emitter, the $\mathrm{K}-40$ in the matrix of the KCFC disk precludes the use of beta proportional counting for the determination of Cs-137 activity.

\section{RAD DISK CHARACTERIZATION STUDIES}

\section{Tc RAD Disk Tests}

Initial tests showed that greater than $95 \%$ of the Tc-99 was removed from spiked one liter solutions of DI water, unfiltered river water or seawater by passing the solution through the TC RAD disk. The flow rate tested initially was $5 \mathrm{~mL} /$ minute and later about $30 \mathrm{~mL} /$ minute. The effect of flow rate was evaluated by pumping spiked DI water through the Tc RAD disk using a peristaltic pump. The $95 \%+$ extraction efficiency was consistent at flow rates up to $100 \mathrm{~mL}$ per minute (Figure 1) using a solution volume of up to 8 liters. Disk were loaded with as much as 5000 pCi of Tc-99, later with up to $15 \mathrm{nCi}$ of Tc-99, with no apparent breakthrough. In a later test two Tc RAD disks were placed in series. Using spiked filtered river water, less than $0.5 \%$ of the Tc- 99 was found on the backup disk for a sample volume of up to 10 liters pumped at a flow rate of $10 \mathrm{~mL} /$ minute (Table 1 ).

$3 \mathrm{M}$ Corp. recommends that samples should be filtered prior to passing through the Empore ${ }^{\mathrm{TM}}$ disks to prevent clogging, as the effective pore size of the disks is about 0.1-0.2 micron. We were usually able to pass one liter of unfiltered river water through the Empore ${ }^{\mathrm{TM}}$ disks or several liters of filtered water. The next series of studies therefore examined the effect of pumping large volumes of unfiltered water through the disks. Unfiltered river water was collected from Steel Creek on the SRS. With no filtration only 1.2-1.75 liters of river water were able to pass through the disk prior to 
complete plugging. Using a graded Whatman filter, with a nominal pore size of 10 to 1 micron, 2.3 3.1 liters of coarsely filtered water was passed through the disk prior to clogging.

A study was next designed whereby the Steel Creek water was passed through filters of different pore sizes prior to passing through the Empore ${ }^{\mathrm{TM}}$ disk. Gelman Supror filters with pore sizes of $0.1,0.2,0.45$ and 0.8 micron were used for this test. The unfiltered creek water was first passed through a graded Whatman filter, then a Gelman filter, then through the Empore ${ }^{\mathrm{TM}}$ disk. Only 1.2 liters of water were able to pass through the $0.1 \mu$ Gelman filter, similarly to the previous test using no filtration. Only 2.8 liters of water were able to pass through the $0.2 \mu$ disk combination, and 3.5 liters of water (the maximum tested here) through the 0.45 and $0.8 \mu$ filters. In all cases the extraction efficiency of Tc-99 from solution was greater than $95 \%$ as determined by liquid scintillation counting of the treated solution. The particle filters and Tc RAD disks were all also counted by liquid scintillation spectrometry. For the smaller particle sizes, 0.1 and $0.2 \mu$, approximately $55 \%$ and $20 \%$, respectively, of the Tc-99 activity was found on the Gelman particle filters rather than the Empore ${ }^{\mathrm{TM}}$ disk. This was reduced to less than $15 \%$ of the Tc-99 on the 0.45 and $0.8 \mu$ particle filters, with the remaining $85 \%+$ on the Empore ${ }^{\mathrm{TM}}$ disk.

Another test completed evaluated the best counting method of the Tc-99 collected on the Tc RAD disk. The 3M technical data sheet on the Tc RAD disk suggests either gas flow beta proportional counting or liquid scintillation spectrometry are suitable for activity determinations. Several disks were prepared by passing spiked DI water or spiked unfiltered river water through Tc RAD disks. Half of the disks were counted by beta proportional counting while the other half were counted by liquid scintillation spectrometry. The disks counted by liquid scintillation spectrometry 
were not dried prior to addition of the cocktail; the disks counted by beta proportional spectrometry were dried at $70^{\circ} \mathrm{C}$ for 15 minutes prior to counting.

The counting efficiency for the DI water disks by liquid scintillation was $68-70 \%$, however the counting efficiency of the river water samples by liquid scintillation was only $6-9 \%$. The unfiltered river water disks did have significant color due to particles removed by the disk, possibly quenching the beta counting efficiency. All disks, DI or river water, counted by beta proportional counting averaged $43.6 \pm 3.1 \%$ counting efficiency. In a separate study an average counting efficiency of $45.0 \pm 10.3 \%$ was calculated for DI and unfiltered river water samples. The larger error in the second set of data was due to the spike activity being less than $4.5 \mathrm{pCi}$ in all cases, which is near the detection limit of the detectors.

We next designed a study to look at the discrimination of the Tc RAD disk against other beta emitting radionuclides which may interfere in the beta proportional counting of Tc-99. Deionized water (one liter) was spiked with either $330 \mathrm{pCi}$ of Cs-137, $1400 \mathrm{pCi}$ of C-14 or $400 \mathrm{pCi}$ of Sr-90. The water was passed through the Tc RAD disk at a flow rate of $30 \mathrm{~mL} /$ minute. The disks were then counted by beta proportional counting; any counts above the detector background would be attributable to the contaminant added. The decontamination factor for the C-14 and Cs- 137 was greater than $99.9 \%$. A small number of counts above background were observed for the solution containing the Sr-90, implying less than $2 \%$ retention of Sr by the Tc RAD disk.

Another test completed on the Tc RAD disks evaluated the effect of water volume passed through the disk versus counting efficiency. A concern was that at higher sample volumes some of the adsorbed Tc- 99 might be embedded further in the disk thereby decreasing the counting efficiency by beta proportional techniques. Sample volumes from 1-10 liters were all spiked with the same amount 
of Tc-99. The sample solutions were passed through the Tc RAD disks at a flow rate of about 20 $\mathrm{mL} /$ minute. After drying, the disks were counted by beta proportional counting. As seen in Table 2 the count rate was constant over the various volumes processed indicating that the Tc-99 counting efficiency was not effected by volume of water processed up to ten liters.

\section{Sr RAD Disk Tests}

The other commercially available Empore ${ }^{\mathrm{TM}} \mathrm{RAD}$ disk evaluated was the Sr RAD disk. $3 \mathrm{M}$ Corp. recommends pretreatment of the Sr RAD disk with methanol and 2M nitric acid prior to passing the sample through the disk. They also recommend that the sample be acidified to $2 \mathrm{M}$ with nitric acid prior to extraction. For simplified processing the feasibility of running the sample with no pretreatment of the disk, nor acidification of the samples, prior to extraction was tested. Solutions of DI water and unfiltered river water were spiked with Sr-90; no additional acid was added. One set of each matrix was passed through the Sr RAD disk with no pretreatment of the disk. The others were passed through the $\mathrm{Sr}$ RAD disk after treatment with $2 \mathrm{~mL}$ of methanol and $20 \mathrm{~mL}$ of $2 \mathrm{M}$ nitric acid, following the manufactures instructions. After the solutions had passed through the disk, they were all treated identically, with a $20 \mathrm{~mL} 2 \mathrm{M}$ nitric acid wash and drying at $70^{\circ} \mathrm{C}$ for 15 minutes. The count rate of the two samples passed through the untreated disks was $57.95 \mathrm{cpm}$ for the DI water and 58.70 $\mathrm{cpm}$ for the unfiltered river water, as opposed to 49.20 and $53.90 \mathrm{cpm}$, respectively, for the treated disks. The untreated disks gave a slightly higher count rate than the treated disks; the difference between the DI and river water is not significant. Based on these results the Sr RAD disk was not pretreated in later studies.

Based on the Tc-99 counting method experiment discussed above beta proportional counting was also used for the Sr RAD disks. Therefore it was necessary to determine the decontamination 
factor by the Sr RAD disk for other beta emitting radionuclides. As for the Tc RAD disks above, one liter samples of DI water were spiked with C-14 (1400 pCi), Cs-137 (130 pCi) or Tc-99 (1700 pCi). The solutions were passed through a Sr RAD disk and then counted by beta proportional spectrometry. The calculated decontamination factor for TC- 99 and C-14 was over $99.98 \%$ but only $98.5 \%$ for Cs-137; less than $2 \%$ of the Cs- 137 was retained by the Sr RAD disk.

In some of the early tests with the Sr RAD disk unexpectedly low and variable count rates on the disk were noted. Discussions with $3 \mathrm{M}$ Corp. technical support indicated they had also noted this effect when the disk was not completely dry prior to counting. During the initial tests; which resulted in the low count rates, the $\mathrm{Sr} \mathrm{RAD}$ disk was dried at $70^{\circ} \mathrm{C}$ for 15 minutes immediately after processing. Later the oven drying step was delayed, allowing the disks to air dry for a few hours before placing them in the oven at $70^{\circ} \mathrm{C}$ for 20 minutes. After this slight modification the inconsistent results were no longer observed.

The next test used spiked unfiltered river water. Sample sizes were varied as shown in Table 3, with all samples receiving the same amount of Sr-90 spike. The Sr RAD disk clogged after only 700 $\mathrm{mL}$ of the one liter solution had been passed through the disk; the result in Table 3 has been corrected for this. The two liter sample finished overnight, thus had air pulled through the disk for an undetermined amount of time prior to the acid rinse and counting. As shown in Table 3, the count rates centered around the expected $55 \mathrm{cpm}$. The only anomaly is the two liter sample count rate of 35 $\mathrm{cpm}$. The four liter sample count rate also seems a bit lower than the other samples, however not significantly. In a later test, variable sample volumes of filtered river water were spiked with Sr-90. The solutions were passed through two $\mathrm{Sr} \mathrm{RAD}$ disks in series at $10 \mathrm{~mL}$ minute. For up to five liters 
there was no apparent breakthrough of Sr-90 to the second disk; $11 \%$ of the Sr-90 was on the second disk for the 7.5 liter sample and $20 \%$ for the 10 liter sample (Table 1 ).

Cs RAD Disk

The first test of the Cs RAD disk consisted of passing one liter of spiked DI water or spiked river water through the Empore ${ }^{\mathrm{TM}}$ disk at a flow rate of $5 \mathrm{~mL} /$ minute. Approximately $60 \mathrm{pCi}$ of Cs137 was added to each solution. The disks and the processed water were counted by gamma spectrometry to determine the uptake efficiency. No Cs-137 was detected in either aliquot of the processed water. The calculated activity of the disk which had been used to extract the Cs-137 from the DI water and the river water was $64 \pm 4 \mathrm{pCi}$ and $61 \pm 4 \mathrm{pCi}$, respectively, indicating a quantitative extraction by the Cs RAD disk under these conditions.

The effect of $\mathrm{pH}$ on the uptake efficiency of the Cs RAD disk was next tested. Five one liter solutions of river water were spiked with $65 \mathrm{pCi}$ of Cs-137. The solutions were then adjusted to a pH of $2,4,6,8$ or 10 using nitric acid and sodium hydroxide. The solutions were then passed through a Cs RAD disk at a flow rate of 0.5 liter per hour (slightly over $8 \mathrm{~mL} /$ minute). As seen in Table 4 , solution $\mathrm{pH}$ had no effect on extraction efficiency. A test to determine the decontamination factor of the Cs RAD disk against some other beta emitting radionuclides was also performed. Carbon-14 and Tc- 99 were found to have a decontamination factor from Cs- 137 of greater than $99.9 \% .15-20 \%$ of the $\mathrm{Sr}-90$ contaminant was retained by the Cs RAD disk. This is not surprising as the KCFC is not as selective of an absorbent as some of the other absorbents used in the Empore ${ }^{\mathrm{TM}}$ technology.

The effect of sample volume on the extraction efficiency was tested by passing various volumes of $0.45 \mu$ filtered river water, spiked with Cs-137, through two Cs RAD disks in series. For sample 
volumes up to five liters no Cs-137 was detected on the backup disk. Even up to 10 liter sample sizes, only a few percent of the Cs-137 was detected on the backup disk (Table 1).

\section{FUTURE WORK}

Based on these results a study was conducted testing the applicability of these solid phase extraction disks for field use. An automated field sampler was developed to collect and process river water through the RAD disks in the field, thereby eliminating the need for any chemical separations to be performed in the lab. The results of the field study are summarized in Beals, et al.?

\section{SUMMARY}

Several solid phase extraction materials made by $3 \mathrm{M}$ Corp. were tested for the extraction of selected radionuclides from aqueous solutions. The Tc RAD disk was found to be quantitative for the extraction of Tc-99 from DI water, river water or seawater, at flow rates up to $100 \mathrm{~mL} /$ minutes.

Samples of up to 10 liters were processed through a single disk, collecting as much as $15 \mathrm{nCi}$ of Tc-99, with less than $1 \%$ breakthrough. Beta proportional counting was found to be the preferred method of quantification due to quenching of the signal by color from filtered particles when using liquid scintillation spectrometry. Decontamination from other beta emitting radionuclides (C-14, Sr-90 and Cs-137) was found to be greater than $99.9 \%$ except for Sr-90, of which less than $2 \%$ was retained by the Tc RAD disk.

Quantitative extractions of Sr-90 were accomplished using the Sr RAD disk on samples of up to five liters, with no pretreatment of the disk, and without acidification of the sample. Counting was again by beta proportional spectrometry, however, the disk must be completely dry prior to counting to 
avoid biasing results low. Decontamination from other beta emitting radionuclides (C-14, Tc-99 and Cs-137) was found to be greater than $99.9 \%$ except for Cs- 137 , of which less than $2 \%$ was retained by the Sr RAD disk.

Quantitative extractions of $\mathrm{Cs}-137$ were also accomplished from aqueous solutions, using a test material containing KCFC. Sample pH and sample volume up to 10 liters had little effect on extraction efficiency. It was found that $15-20 \%$ of added Sr-90 was retained by the Cs RAD disk. Counting of the Cs-137 was by gamma spectrometry thus decontamination from other beta emitters was not as important for the Cs RAD disk as for the Tc RAD and Sr RAD disks counted by beta proportional counting.

Using the results obtained during this study, laboratory based methods for the analysis of Tc99, Sr-90 and Cs-137 should be significantly improved. Typical sample volumes currently used are only one liter; several liters of sample can be processed through the Empore ${ }^{\mathrm{TM}} \mathrm{RAD}$ disks enabling lower detection limits to be obtained. Current methods of analysis for Tc-99 and Sr-90 require extensive chemical separation which would be eliminated using the SPE technique. Overall, these RAD disks appear to meet the criteria of providing faster, better and cheaper results for aqueous radiochemical determinations.

\section{ACKNOWLEDGMENTS}

The authors wish to thank $3 \mathrm{M}$ Corp. for preparing and supplying the experimental RAD disks used in this study, and especially Craig Markell and Peter Ellefson of 3M Corp. for their technical help. We also thank Brian Crandall of the Environmental Monitoring Section of the SRS for the use of his 
counting room detectors. The information in this document was produced during activities performed under contract No. DE-AC09-89SR18035 for the U.S. Department of Energy.

\section{REFERENCES}

1. Hagen, D.F., C.G. Markell, G.. Schmitt and D.D. Blevins, "Membrane Approach to Solid-Phase Extractions," Analytica Chimica Acta, v. 236, p. 157-164, 1990.

2. Beals, D.M., "Determination of Technetium-99 in Aqueous Samples by Isotope Dilution Inductively Coupled Plasma Mass Spectrometry," Journal of Radioanalytical and Nuclear Chemistry, v.204, p. 253-263, 1996.

3. Bibler, J.P., "Year-End Report for UST: Cesium Extraction Testing Project DOE/DT\&E, TTP No. SR1-03-20-01 (U)," Westinghouse Savannah River Company, WSRC-RP-94-146, 1994.

4. Boni, A.L., "Rapid Ion Exchange Analysis of Radiocesium in Milk, Urine, Sea Water and Environmental Samples," Analytical Chemistry, v. 38, p. 89-92, 1966.

5. Godoy, J.M., J.R.D. Guimaraes and Z.L. Carvalho, "Cs-137 Pre-concentration from Water Samples Using a Prussian Blue Impregnated Ion-Exchanger," Journal of Environmental Radioactivity, v. 20, p. 213-219, 1993.

6. Beals, D.M., J.P. Bibler and D.A. Brooks, "Extension of Studies with $3 \mathrm{M} \mathrm{Empore}{ }^{\mathrm{TM}}$ and Selentec MAG*SEP ${ }^{\text {SM }}$ Technologies for Improved Radionuclide Field Sampling, TTP No, SR-16C343

(U)," Westinghouse Savannah River Company, WSRC-TR-96-0206, 1996.

7. Beals, D.M., W.G. Britt, P. Fledderman, B.S. Crandall, R.E. Setter, R. Fiedler, C.G. Markell and K.M. Hoffmann, "An Automated Field Sampler Incorporating Solid Phase Extraction Disks for the 
Quantification of Radionuclides in Surface Streams." Presented at PittCon 97, Atlanta GA; in preparation for Journal of Field Analytical Chemistry and Technology.

\section{LIST OF FIGURES}

Figure 1: Flow Rate versus Tc-99 Retention by' Empore ${ }^{\mathrm{TM}}$ disk

\section{LIST OF TABLES}

Table 1: Sample volume versus extraction efficiency of Empore ${ }^{\mathrm{M}} \mathrm{RAD}$ disks

Table 2: Tc-99 counting efficiency versus sample volume processed

Table 3: Sr-90 counting efficiency versus sample volume processed

Table 4: pH Effect on the Extraction Efficiency of Cs-137 from River Water 


$$
\omega S R C-m S-96-0680
$$

Figure 1: Flow Rate versus Tc-99 Retention by Empore Disk

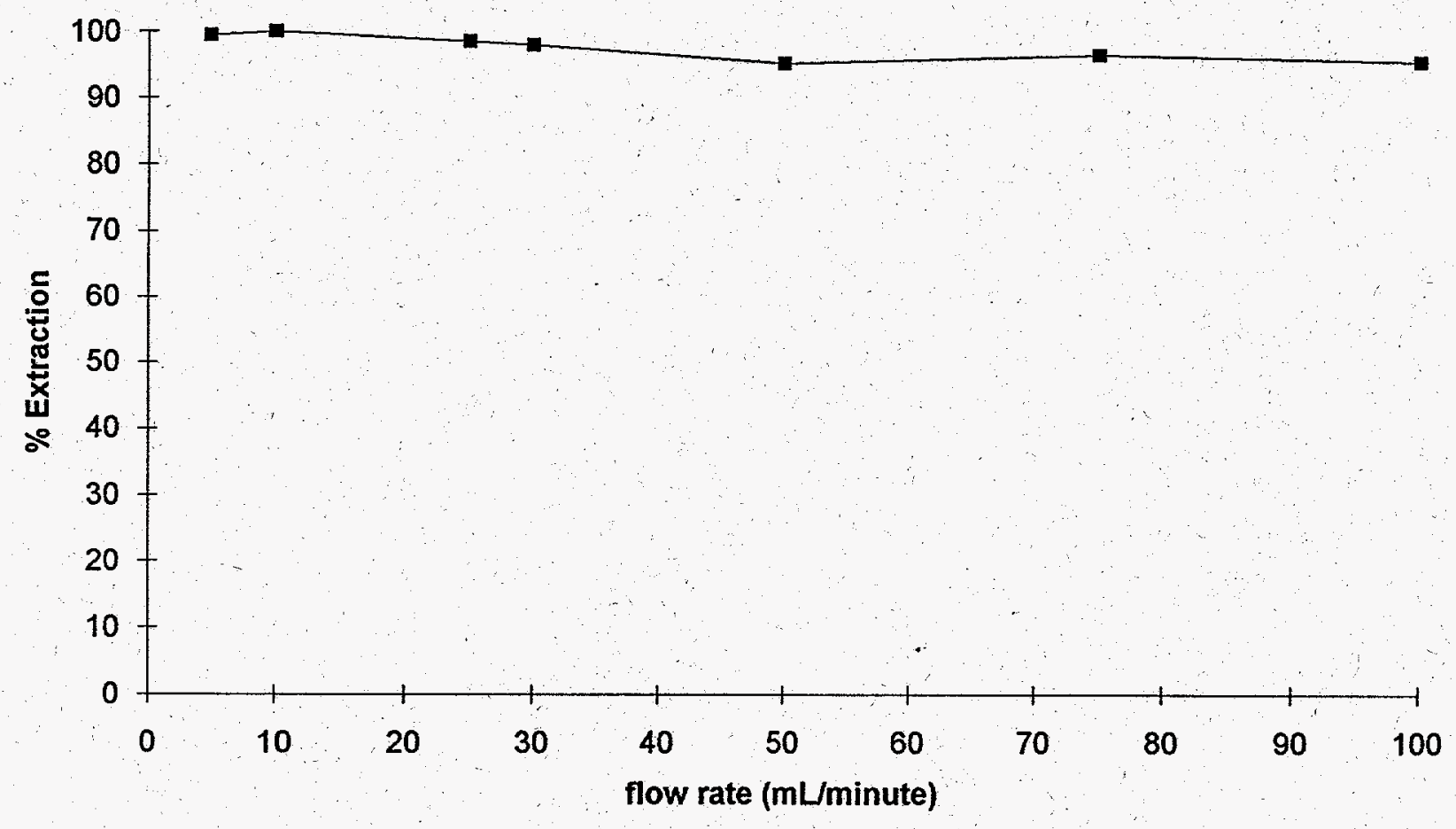


Table 1: Sample Volume versus Extraction Efficiency of Empore RAD Disks

\begin{tabular}{|c|c|c|c|c|c|c|c|c|c|}
\hline $\begin{array}{l}\text { sample volume } \\
\text { (liters) }\end{array}$ & first disk & $\begin{array}{l}\text { Tc-99 (cpm) } \\
\text { second disk }\end{array}$ & $\%$ breakthru & first disk & $\begin{array}{l}\text { Sr-90 (cpm) } \\
\text { second disk }\end{array}$ & $\%$ breakthru & first disk & $\begin{array}{l}\text { Cs-137 (pCi) } \\
\text { second disk }\end{array}$ & $\%$ breakthn \\
\hline 1 & 5392.9 & -0.5 & -0.01 & 67.25 & 0.05 & 0.07 & 58.6 & $<0.6$ & $<1$ \\
\hline 2.5 & 7696.2 & -0.7 & -0.01 & 67.25 & -0.20 & -0.30 & 56.8 & $<0.4$ & $<0.7$ \\
\hline 5 & 11592.9 & 0.2 & 0.00 & 48.80 & 1.05 & 2.11 & 63.0 & 0.4 & 0.58 \\
\hline 7.5 & 7755.9 & 27.9 & 0.36 & 47.75 & 5.90 & 11.00 & 41.9 & 1.2 & 2.83 \\
\hline 10 & 8148.0 & 26.2 & 0.32 & 46.25 & 11.95 & 20.53 & 38.0 & 1.3 & 3.31 \\
\hline
\end{tabular}

Table 2: Tc-99 Counting Efficiency verșus Sample.Volume Processed

\begin{tabular}{ccc}
$\begin{array}{c}\text { sample volume } \\
\text { (liters) }\end{array}$ & $\begin{array}{c}\text { Ta99 } \\
\text { (cpm) }\end{array}$ & $\begin{array}{c}\text { error } \\
\text { (1 sigma) }\end{array}$ \\
\hline 1 & 1182.1 & 7.7 \\
2 & 1319.9 & 8.2 \\
4 & 1178.7 & 7.7 \\
6 & 1240.0 & 7.9 \\
8 & 1203.1 & 7.7 \\
10 & 1270.0 & 8.0
\end{tabular}

Table 3: Sr-90 Counting Efficiency versus Sample Volume Processed

$\begin{array}{ccc}\begin{array}{c}\text { sample volume } \\ \text { (liters) }\end{array} & \begin{array}{c}\text { Sr-90 } \\ \text { (cpm) }\end{array} & \begin{array}{c}\text { error } \\ \text { (1 sigma) }\end{array} \\ 0.05 & 55.9 & 1.7 \\ 0.1 & 58.8 & 1.7 \\ 0.25 & 60.3 & 1.7 \\ 0.5 & 58.2 & 1.7 \\ 1 & 65.9 & 2.2 \\ 2 & 35.1 & 1.3 \\ 4 & 49.2 & 1.6\end{array}$

Table 4: pH Effect on the Extraction Efficiency of Cs-137 from River Water

\begin{tabular}{|c|c|c|}
\hline $\mathrm{pH}$ & $\begin{array}{c}\mathrm{Cs}-137 \\
(\mathrm{pCi})\end{array}$ & $\begin{array}{c}\text { error } \\
\text { (1 sigma) }\end{array}$ \\
\hline 2 & 68.0 & 4.6 \\
\hline 4 & 55.3 & 4.3 \\
\hline 6 & 75.6 & 5.1 \\
\hline 8 & 64.8 & 4.7 \\
\hline 10 & 69.3 & 4.7 \\
\hline
\end{tabular}

